\title{
A single-center experience with service organization for patients with ARFID
}

\author{
Mark Norris ( $\nabla$ mnorris@cheo.on.ca ) \\ Children's Hospital of Eastern Ontario \\ Nicole Obeid \\ Children's Hospital of Eastern Ontario \\ Alexandre Santos \\ The University of British Columbia School of Kinesiology

\section{Darcie Valois} \\ Children's Hospital of Eastern Ontario

\section{Leanna Isserlin} \\ Children's Hospital of Eastern Ontario

\section{Stephen Feder} \\ Children's Hospital of Eastern Ontario

\section{Wendy Spettigue} \\ Children's Hospital of Eastern Ontario
}

\section{Research article}

Keywords: Avoidant restrictive food intake disorder, service organization, pilot study, retrospective cohort study, multidisciplinary, treatment

Posted Date: April 20th, 2020

DOI: https://doi.org/10.21203/rs.3.rs-22008/v1

License: (c) (1) This work is licensed under a Creative Commons Attribution 4.0 International License.

Read Full License 


\section{Abstract \\ Background}

To date, very little research has explored the impact that the newly articulated diagnosis Avoidant restrictive food intake disorder (ARFID) has had on feeding and eating disorder program service organization and delivery. The purpose of this paper is to provide a descriptive overview of a single-center, ARFID-specific pilot clinic that sought to better understand the specific needs of patients with ARFID and gain insight into treatment requirements.

\section{Methods}

A retrospective cohort study was completed on patients with ARFID admitted to a specialized pilot clinic within a tertiary care hospital.

\section{Results}

Over an 18 month period, a total of 31 patients were assessed, with 26 patients completing follow-up assessments. Patients presented with heterogeneous manifestations of ARFID, with treatment plans tailored to meet individual needs at assessment and over the treatment period. A multidisciplinary approach was most often administered, including a combination of administered individual therapy, family therapy, medical monitoring, and prescribed medications. Only $30 \%$ of patients were treated exclusively by therapists on the eating disorder team.

\section{Conclusions}

The experiences gained from this pilot study highlight the need for specialized resources for assessment and treatment of patients with ARFID, the importance of a multidisciplinary approach to treatment, and the necessity of utilization of ARFID-specific measures for program evaluation purposes.

\section{Plain English Summary}

The present study describes an overview of a single-center pilot clinic designed specifically to treat youth with avoidant restrictive food intake disorder (ARFID) in order to better understand the needs of patients with ARFID. Participants were 31 patients with ARFID who were treated with a multidisciplinary approach and had treatment plans tailored to their specific needs. Findings highlight the need for specialized resources for assessment and treatment of patients with ARFID, the importance of a multidisciplinary approach to treatment, and the need for ARFID-specific measures for program evaluation purposes

\section{Background}


Avoidant restrictive food intake disorder (ARFID) is a feeding or eating disturbance introduced in the Diagnostic and Statistical Manual of Mental Disorders Fifth Edition(1) and described in the section titled "Feeding and Eating Disorders". Since its introduction, researchers have sought to better understand and describe ARFID(2-4). Many early studies have reported on patients drawn exclusively from eating disorder (ED) programs and have attempted to understand ARFID in the context of other EDs, including anorexia nervosa (AN)(5-8). Emerging research has suggested that the ARFID diagnosis encapsulates a heterogeneous group of patients with varying (and at times mixed) presentations that may be distinct in etiology $(9,10)$; this includes a proportion of patients with ARFID who present with histories of lengthy preexisting feeding disturbances(3). Compared to patients with other ED diagnoses, multiple studies have found that patients with ARFID are younger than those with AN, more likely to be male, and have higher rates of anxiety (5-7).

For the past 20 years, our pediatric ED team has triaged, assessed, and treated patients with severe EDs as evidenced by medical instability, growth compromise, suicidal ideation in the context of an active ED, or substantial ED symptom impact. Our hospital-based multidisciplinary team of ED healthcare professionals provide treatment in outpatient, inpatient, and day hospital settings. Our team members consist of social workers who provide family-based therapy (FBT), psychologists who provide FBT and various cognitive-based therapies, and psychiatrists who prescribe psychotropic medication and deliver FBT/cognitive-based therapies as required. Dietary and nutritional treatment is provided by registered dietitians, while adolescent health physicians and a nurse practitioner manage medical care and provide general mental health support to patients and families. However, patients with chronic feeding disorders have historically been managed by a variety of other hospital-based specialists from varying disciplines (i.e. occupational therapists, behavioral psychologists, pediatricians, etc.), or in the case of infants and children less than 6 years of age, a dedicated interdisciplinary team of feeding specialists consisting primarily of occupational therapists, psychologists, and a registered dietitian(11). At present, there is no dedicated resource within our region for older children and adolescents with complex feeding presentations that are not linked to underlying body image concerns.

Prior to the introduction of ARFID in the DSM-5, program evaluation data demonstrated the existence of a small cohort of older children and adolescents with restrictive feeding behaviors who lacked body image concerns but exhibited significant restricted intake and medical compromise(6). Given the lack of body image concerns and absence of a diagnosis of an ED such as AN or bulimia nervosa (BN), these patients historically received treatment outside of the ED program by a myriad of healthcare providers within the hospital and greater community, including the consultation-liaison psychiatry team and the gastroenterology team, amongst others. As education and research surrounding ARFID as a new disorder in the DSM-5 increased, so too did the number of requests for formal ED assessments for such patients. This has resulted in a host of operational questions for our ED program as well as the hospital, including those relating to the ability and suitability of patients with ARFID to be managed by our ED program, mechanism of triage, assessment tools, program evaluation metrics, and treatment provision. With funded resources for patients with $\mathrm{AN}$ and $\mathrm{BN}$ already limited within our region, creative discussion 
among healthcare providers has been required to best determine how patients meeting criteria for ARFID can be optimally and efficiently managed.

To better understand the specific needs and gain insight into treatment requirements of patients with ARFID, a dedicated clinic for older children and adolescents meeting criteria for ARFID was piloted at our hospital. This paper provides a descriptive overview of such experience and includes both challenges and knowledge gained throughout the study's timeframe.

\section{Methods}

After careful discussion with key stakeholders across various departments within a tertiary care children's hospital in Ontario, Canada (i.e. Pediatrics and Mental Health) and with the understanding that resources required to staff and support a "new" multi-disciplinary clinic were unavailable, a pilot clinic was initiated. The half-day, bi-weekly ARFID clinic was piloted for approximately 18 months (December 2014 to June 2016) at the hospital, where a senior adolescent medicine physician (MLN) with substantial experience in the assessment and care of patients with EDs as well as those with complex feeding presentations presided over all clinic operations. Referrals were triaged by the ED team; triage acceptance criteria were set as patients age 10 and older with evidence of medical instability (i.e. severe bradycardia, hypotension, or growth compromise) secondary to a feeding disturbance or ED that was not obviously attributable to body image concerns or fear of weight gain. Of note, our triage criteria for medical instability were in line with criteria used by our ED program for referral acceptance.

For patients meeting acceptance criteria into the pilot clinic, all primary assessments were completed by the clinic's overseeing physician. At assessment, a thorough developmental, feeding, nutritional, and psychosocial history was completed using individual and family interviews. Psychological measures using ED-specific questionnaires were utilized in the early months of the clinic but given their limitations and strain on limited psychometric resources, this practice stopped after approximately 4-6 months. Psychological measures were administered thereafter when either concerns regarding the presence of possible AN were raised during or after initial assessment, in cases where substantial mental health distress was present and felt to be interfering with treatment, or if greater clarity regarding the presence of anxiety or mood disorders was required. It should be noted that ARFID-specific measures or inventories were not available during the timeframe that the pilot clinic operated. Laboratory and other diagnostic testing protocols were conducted at assessment on an individualized basis. Treatment was provided to patients on an individual basis. The primary physician liaised with other treatment providers to arrange and provide treatment specific to individuals' needs (e.g. members of the ED team as well as those from other disciplines in the hospital). Follow-up care was provided by the primary physician over the course of the pilot timeframe.

Data pertaining to patients' assessments and treatment trajectories for the duration of this pilot were gathered via a retrospective chart review by members of the research team. In order to provide a narrative of treatments offered and received, we included only patients with data from both assessment and 6/12 
months post-assessment. Data were collected and analyzed using SPSS 24.0. Descriptive and frequency analyses were performed where appropriate. A relatively small sample size precluded any comparison analyses between groups. This study received ethical approval from the hospital's Research Ethics Board.

\section{Results}

Over 18 months, a total of 31 new patients were assessed at the pilot clinic. Of these, follow-up data at one year was available for 26 patients (see Fig. 1). Of the 26 patients, $31 \%(n=8)$ presented with a chief complaint of low appetite or food indifference, $23 \%(n=6)$ presented with an acute history of restriction suggesting that the feeding disturbance was precipitated by a traumatic feeding or life event (i.e. secondary to at least one "aversive experience"), $8 \%(n=2)$ presented with a feeding disturbance attributed primarily to longstanding sensory-based food avoidance (i.e. very severe selective eating), and $38 \%(n=10)$ presented with a mixed presentation (at least two of the previously described presentations were felt to be contributing).

Demographic information as well as illness and treatment specific indicators are provided in Table 1. The overwhelming majority of patients $(92 \%, n=24)$ were low weight at time of initial assessment. Thirty five percent of patients and/or caregivers identified sensory issues related to feeding as a barrier to normalized eating at first assessment. Self-reported length of illness was substantially shorter in those with histories of acute restriction secondary to an aversive experience as compared to other presentations. Mental health comorbidity was high in all groups (Table 2). While not the case for youth with low appetite/food indifference, more than $50 \%$ of patients in each of the remaining and mixed groups had a concurrent anxiety disorder. Twenty seven percent of assessed patients had a diagnosis of autism or autism-spectrum disorder.

Treatment plans varied according to the chief presenting concerns and the disposition of the patient at time of assessment (i.e. outpatient vs. inpatient setting). Similar to family-based treatment principles for AN, primary treatment goals of the adolescent medicine physician focused on weight rehabilitation when malnutrition was present, as well as empowering families to take control of their child's nutrition and increase calories as required. Treatment plans evolved according to identified needs of the patients (see Table 3). Although not formally tracked, the frequency of clinic visits with the treating physician varied in accordance with treatment progress, such as the degree of medical compromise and the progression of weight gain. While half of patients with acute restriction due to an aversive experience required hospitalization due to medical instability or absolute food refusal, only $15 \%$ of the remaining patients (including those with mixed presentations) were hospitalized at any point during treatment. Depending on case-specific needs, individual/family therapists were recommended when assistance was required achieving weight restoration, or if mental health morbidity or oral-motor sensitivities were felt to be impacting feeding-specific goals. Family therapy was most likely to be recommended for those with aversive/acute presentations, whereas varying individual psychological therapies (e.g. cognitive behavioral therapy (CBT) with exposure, behavioral therapies) were utilized in other presentations, such 
as sensory sensitivity. Only $30 \%(n=8)$ of patients received psychological therapy exclusively by ED therapists.

The need for clinical reassessment of treatment goals by the treating physician varied and included such changes as: a psychological or behavioral therapy was initiated, support by a multi-disciplinary member (i.e. a dietitian) was required, a medication was prescribed, or a change in treatment setting occurred (i.e. patients required admission to hospital or were discharged from hospital; see Table 4). Regarding rates of weight restoration, just over half $(54 \%, n=14)$ of patients were weight restored by six months postassessment. Of note, the median percentage of treatment goal weight (TGW) attained ranged between 96-100\% across all cohorts at the time of discharge from treatment (Table 1). Those with mixed presentations were proportionately more likely to still be engaged with clinical services at one year after initial assessment.

\section{Discussion}

The objective of this study was to gather knowledge and insight through a single-center pilot initiative of a clinic for youth with ARFID. Our experience highlighted several key findings that have helped our hospital better understand how resources can be aligned to best meet the needs of such patients. First, despite the very stringent inclusion criteria set for this initiative, a total of 31 patients were assessed, suggesting a strong need for a clinic or service within our hospital that serves older children and adolescents with complex feeding presentations.

Although our inclusion criteria for acceptance into the pilot clinic were purposefully narrow, our justification was that the high medical needs of patients assessed would almost certainly have resulted in assessment by at least one hospital-based physician or service during the timeframe that the pilot ran. Regarding inclusion criteria, this experience highlighted an important lesson learned relating to our omission of markers of impairment on our triage form. By only accepting referrals with demonstrated evidence of medical instability, we excluded patients that met criteria for ARFID solely on the grounds of psychosocial impairment, even when severe. This has relevance given recent study findings that suggest that the patients with ARFID who are included based primarily on the criterion of psychosocial impairment are often normal weight(12). Although one of our purposes in establishing this pilot clinic was to better understand the scope and complexity of issues that patients with ARFID might present with, our decision to limit inclusion criteria introduced bias into our results. This would be an important consideration for future program and resource planning. Further research is also required to clarify how the degree of psychosocial impairment affects illness presentation as well as response to treatment in each of the types of presentations of ARFID.

Although each of the proposed 'motivations' for feeding disturbances outlined in the DSM-5 was represented in this pilot endeavor, those presenting with primary feeding challenges related to the sensory domain (limited-variety type) were fewer in number as compared to those with other recognized 
presentations. Our own research has suggested that those with sensory-based food avoidance (i.e. extremely selective eaters) are less likely to present with markers of medical instability and as such, would have been less likely to meet inclusion criteria for this pilot initiative(10). Although ED-program specific research in this area remains in its infancy, the hypothesis that those with feeding issues related primarily to sensory difficulties have longer illness courses and lower rates of medical instability as compared to other ARFID presentations is supported by our current findings and a previous study with a larger sample size(10).

Conceptually, if food restriction in ARFID results from lack of appetite, difficulties with sensory characteristics of food, and/or fears related to the act of eating (or combinations of these characteristics), assessment tools and treatment plans need to be able to clearly delineate these specific etiologies, help confirm diagnoses, and meet specific patient treatment needs(13). Given current resource constraints as well as our ED program's very specific approach to the assessment and treatment of patients with severe EDs such as $\mathrm{AN}$ and $\mathrm{BN}$, it was unrealistic to assume that treatment options available for patients with AN and BN could cover the breadth of needs of patients with ARFID. Our data supported this assumption, with only $30 \%$ of patients exclusively receiving therapy by ED team members. Indeed, the issue of heterogeneous treatment requirements for patients with ARFID was regularly noted for those with mixed presentations. Not uncommonly, patients in this cohort required care by multiple team members across different disciplines, highlighting the necessity of a treatment framework that can adapt to meet the varying needs of patients, as opposed to a "one size fits all" approach. Of interest, a recent systematic review of interventions for severe feeding disorders suggested an intensive multidisciplinary team approach and is in keeping with a best-practice model(14).

In addition to these considerations, another lesson learned from the study relates to the limited capacity of existing programs to accommodate patients with ARFID. Despite strict inclusion criteria, and a limited assessment window, the pilot initiative resulted in 31 assessments in 18 months, or the equivalent of 21 new patients annually. This represents approximately one third of annual assessments typically completed by our ED program. Given that patients with ARFID are increasingly recognized as requiring prolonged treatment courses, considerations that relate to any existing program's capacity to accept new referrals cannot be understated. As our hospital currently lacks a dedicated feeding team for children over the age of 6 years, the described pilot helped bring attention to existing treatment gaps (i.e. sensoryspecific feeding interventions for older children and adolescents). It also highlighted the finding that a proportion of patients with ARFID benefit from feeding-specific interventions that involve support, education and gradual exposure to foods deemed unsafe or unappealing. Depending on the specific resources available within institutions, this treatment could be provided outside of the ED team (as was typically the case in this initiative) or by ED team members assuming additional training and support could be provided.

Regarding the assessment procedure, in keeping with the findings of Cooney and colleagues(15), our ED program's psychometric measures were not specific for making a diagnosis of ARFID, and given resource constraints, the practice was stopped. Moving forward, should a coordinated program of evaluation be re- 
established, a number of inventories and measures are now available that would help practitioners better track progress of outcome variables specific to ARFID as treatment progresses $(16,17)$.

In keeping with previous studies, we demonstrated that patients accepted into this pilot study exhibited high rates of mental health comorbidity, the most notable of which was anxiety. This finding provides additional support for the utility of therapies that are mindful of the mental health needs of patients and that provide support and psychoeducation to families. As is the case in the treatment of AN, our initial emphasis in underweight patients with ARFID has been to renourish patients to an optimal TGW, with focus on improved nutritional intake and weight gain during the primary phase of treatment. A recent case series highlighted the multi-disciplinary team approach involving family therapy and cognitive behavioral therapy for the care of patients with varying ARFID presentations(18).

Patients that required inpatient hospitalization were discharged to outpatient services with continued emphasis on renourishment and treatment of the feeding disturbance. Although an emerging body of literature has suggested that patients with ARFID can be treated using day hospital models, our existing day treatment program (DTP) was designed for treatment of patients with AN and BN (e.g. includes groups focused on body image). We suggest that further research that explores the utility and optimization of DTP content would help programs such as our own to understand whether modifications should be considered to make the treatment suitable for patients with ARFID.

This pilot initiative also provided some insight into the use of pharmacotherapy as an adjunctive treatment for patients with ARIFD. We demonstrated that patients across all subtypes were treated with a myriad of different medications from different classes. As an example, atypical antipsychotics (such as olanzapine) were offered to half of the patients presenting with aversive presentations. Although not empirically studied herein, this treatment approach targeted anxiety in an attempt to allow the patient greater ability to accept foods deemed triggering or unsafe (e.g. something that they feared they would choke on, or would cause them to vomit) or to decrease the overall intensity of internal distress. To date, the evidence examining olanzapine efficacy in patients with ARFID has been limited to two case series $(18,19)$. The use of appetite stimulants (such as cyproheptadine) also requires further study in this population, in order to better understand how these agents might serve to promote nutritional intake and to what extent illness presentation predicts response(20).

Our outcomes at the six-month mark suggest that similar to those with other EDs, many patients with ARFID require intensive services for prolonged periods. Those with mixed presentations required care by many multidisciplinary team members, including other medical subspecialists. Although our small sample size precludes statistical comparisons, those with mixed presentations had higher rates of weight restoration at 6 months compared to patients with a single primary influence (e.g. selective eating or low appetite) for the feeding disturbance. It is difficult to know what, if any factors influenced this specific finding, although these patients had more diversified treatment teams. As the number of empirically studied treatments for patients with ARFID evolves, it is likely that researchers will gain a better 
understanding of how treatments should be dosed and delivered in order to optimize outcomes across respective presentations.

As noted throughout the discussion, this pilot study was not without limitations. We embarked on this initiative as a means of better understanding the types and presentations of patients that might present to a dedicated clinic for patients with severe ARFID. By limiting our inclusion criteria and the study timeframe, we introduced referral bias and limited our total sample. Our results are retrospective and given the lack of diagnostic instruments available at the time of the pilot, standardized and validated measures specific to ARFID were not utilized. Strengths of the study included the fact that a single provider with expertise in ARFID and complex feeding disorders triaged, assessed, and coordinated care for all patients. Also, a consistent interview template was used for all assessments. Remarkably, despite a lack of coordinated programming, almost all patients were weight restored by the time treatment concluded. Although not formally evaluated, chart review suggested that treating the subset of patients with sensory-specific feeding issues (i.e. limited-variety type) provided challenges given gaps within the hospital for services specific to the needs of this population. In cases where family-based therapy principles were applied to patients with sensory-specific issues, food selectivity often persisted despite success with weight gain. Most typically, these patients presented with long-standing histories of restrictive eating, dating back to infancy. The lack of a validated instrument that assessed severity and tracked progress in this particular area is another limitation of the study.

This pilot project provided additional evidence that there is an unmet need for coordinated services focused on the assessment and treatment of children and adolescents with complex feeding presentations. Although not formally tracked, a number of referrals were denied because they did not meet our strictly defined inclusion criteria. The small clinic ran at capacity throughout its 18-month operational window. Although we have demonstrated the effectiveness of ED team members to provide treatment to at least a proportion of patients (e.g. using family-based therapy and cognitive therapy), additional resources and the input of other multi-disciplinary care providers outside of the ED team were often required.

\section{Conclusions}

Moving forward, it will be important for health providers who assess and provide care to patients with ARFID to have an understanding of the various presentations that encapsulate this heterogeneous disorder and to utilize assessment tools and targeted treatments that best match individualized patient needs. As evidence becomes available, treatment pathways and protocols can be developed that draw upon existing expertise (likely within and outside of ED teams) to serve patients with diverse presentations. As noted in Sharpe \& Stubbs' recent commentary(21), tailored feeding interventions need to account for the behavioral, psychological, organic, oral-motor, and dietary concerns that often co-exist in complex feeding and eating disturbances. Our experience with this project has resulted in a better understanding of the need for hospitals to provide a range of specialized, multi-disciplinary resources directed at the triage, assessment, and treatment of youth with ARFID who may present in a variety of 
ways. This experience also helped highlight specific gaps in services in our hospital, which in turn has resulted in a better multi-disciplinary approach to service coordination for patients (aged 10-17 years) with complex feeding presentations. Although there is much to learn, we remain collectively committed to understanding how precious healthcare resources can be best organized and adapted to meet the needs of patients with complex feeding concerns across the pediatric lifespan.

\section{Abbreviations}

ARFID - Avoidant restrictive food intake disorder

ED - eating disorder

AN - anorexia nervosa

FBT - family-based therapy

BN - bulimia nervosa

CBT - cognitive behavioral therapy

TGW - treatment goal weight

DTP - day treatment program

\section{Declarations}

Funding details: This work was not supported by a source of funding.

Disclosure statement: No potential conflicts of interest were reported by the authors.

Ethics Approval: This study received ethical approval from the Children's Hospital of Eastern Ontario's Research Ethics Board (CHEOREB\#15/98X).

Consent for Publication: NA

Availability of Data: The datasets used and/or analysed during the current study are available from the corresponding author on reasonable request

Competing interests: NA

\section{Author's Contributions:}

MN conceptualized the study, supervised data collection, and wrote the paper.

NO helped conceptualize the study, supervised data collection, and provided feedback on the paper. 
AS completed data collection, helped write and provide feedback on the paper.

DV completed data collection, helped write the paper.

LI helped conceptualize the study, reviewed results, and helped write the paper.

SF helped conceptualize the paper, reviewed results, and helped write the paper.

WS helped conceptualize the paper, reviewed results, and helped write the paper.

All authors read and approved the final manuscript

Acknowledgements: NA

\section{References}

1. American Psychiatric Association. Diagnostic and statistical manual of mental disorders (DSM-5®). American Psychiatric Pub; 2013.

2. Fisher MM, Rosen DS, Ornstein RM, Mammel KA, Katzman DK, Rome ES, et al. Characteristics of avoidant/restrictive food intake disorder in children and adolescents: a "new disorder" in DSM-5. J Adolesc Heal. 2014;55(1):49-52.

3. Katzman DK, Norris ML, Zucker N. Avoidant restrictive food intake disorder. Psychiatr Clin. 2019;42(1):45-57.

4. Norris ML, Katzman DK. Change is never easy, but it is possible: reflections on avoidant/restrictive food intake disorder two years after its introduction in the DSM-5. J Adolesc Heal. 2015;57(1):8-9.

5. Nicely TA, Lane-Loney S, Masciulli E, Hollenbeak CS, Ornstein RM. Prevalence and characteristics of avoidant/restrictive food intake disorder in a cohort of young patients in day treatment for eating disorders. J Eat Disord. 2014;2(1):21.

6. Norris ML, Robinson A, Obeid N, Harrison M, Spettigue W, Henderson K. Exploring avoidant/restrictive food intake disorder in eating disordered patients: a descriptive study. Int J Eat Disord. 2014;47(5):495-9.

7. Ornstein RM, Essayli JH, Nicely TA, Masciulli E, Lane-Loney S. Treatment of avoidant/restrictive food intake disorder in a cohort of young patients in a partial hospitalization program for eating disorders. Int J Eat Disord. 2017;50(9):1067-74.

8. Strandjord SE, Sieke EH, Richmond M, Rome ES. Avoidant/restrictive food intake disorder: illness and hospital course in patients hospitalized for nutritional insufficiency. J Adolesc Heal. 2015;57(6):6738.

9. Duncombe Lowe K, Barnes TL, Martell C, Keery H, Eckhardt S, Peterson CB, et al. Youth with avoidant/restrictive food intake disorder: examining differences by age, weight status, and symptom duration. Nutrients. 2019;11(8):1955. 
10. Norris ML, Spettigue W, Hammond NG, Katzman DK, Zucker N, Yelle K, et al. Building evidence for the use of descriptive subtypes in youth with avoidant restrictive food intake disorder. Int J Eat Disord. 2018;51(2):170-3.

11. Owen C, Ziebell L, Lessard C, Churcher E, Bourget V, Villenueve H. Interprofessional group intervention for parents of children age 3 and younger with feeding difficulties: pilot program evaluation. Nutr Clin Pract. 2012;27(1):129-35.

12. Becker KR, Keshishian AC, Liebman RE, Coniglio KA, Wang SB, Franko DL, et al. Impact of expanded diagnostic criteria for avoidant/restrictive food intake disorder on clinical comparisons with anorexia nervosa. Int J Eat Disord. 2019;52(3):230-8.

13. Thomas JJ, Lawson EA, Micali N, Misra M, Deckersbach T, Eddy KT. Avoidant/restrictive food intake disorder: a three-dimensional model of neurobiology with implications for etiology and treatment. Curr Psychiatry Rep. 2017;19(8):54.

14. Sharp WG, Volkert VM, Scahill L, McCracken CE, McElhanon B. A systematic review and metaanalysis of intensive multidisciplinary intervention for pediatric feeding disorders: how standard is the standard of care? J Pediatr. 2017;181:116-24.

15. Cooney M, Lieberman M, Guimond T, Katzman DK. Clinical and psychological features of children and adolescents diagnosed with avoidant/restrictive food intake disorder in a tertiary care eating disorder program. J Adolesc Heal. 2017;60(2):46.

16. Bryant-Waugh R, Micali N, Cooke L, Lawson EA, Eddy KT, Thomas JJ. Development of the Pica, ARFID, and Rumination Disorder Interview, a multi-informant, semi-structured interview of feeding disorders across the lifespan: A pilot study for ages 10-22. Int J Eat Disord. 2019;52(4):378-87.

17. Zickgraf HF, Ellis JM. Initial validation of the nine item avoidant/restrictive food intake disorder screen (NIAS): a measure of three restrictive eating patterns. Appetite. 2018;123:32-42.

18. Spettigue W, Norris ML, Santos A, Obeid N. Treatment of children and adolescents with avoidant/restrictive food intake disorder: a case series examining the feasibility of family therapy and adjunctive treatments. J Eat Disord. 2018;6(1):20.

19. Brewerton TD, D'Agostino M. Adjunctive use of olanzapine in the treatment of avoidant restrictive food intake disorder in children and adolescents in an eating disorders program. J Child Adolesc Psychopharmacol. 2017;27(10):920-2.

20. Harrison ME, Norris ML, Robinson A, Spettigue W, Morrissey M, Isserlin L. Use of cyproheptadine to stimulate appetite and body weight gain: A systematic review. Appetite. 2019.

21. Sharp WG, Stubbs KH. Avoidant/restrictive food intake disorder: A diagnosis at the intersection of feeding and eating disorders necessitating subtype differentiation. Int J Eat Disord. 2019;52(4):398401.

\section{Tables}

Table 1. Demographic and assessment characteristics of pediatric ARFID patients $(n=26)$ admitted to a specialized pilot clinic. 


\begin{tabular}{|c|c|c|c|c|c|c|c|c|}
\hline & \multicolumn{2}{|c|}{$\begin{array}{l}\text { ARFID-Aversive Hx } \\
\qquad(\mathrm{n}=6)\end{array}$} & \multicolumn{2}{|c|}{$\begin{array}{l}\text { ARFID-Limited Variety } \\
\qquad(\mathrm{n}=2)\end{array}$} & \multicolumn{2}{|c|}{$\begin{array}{l}\text { ARFID-Limited Intake } \\
\qquad(\mathrm{n}=8)\end{array}$} & \multicolumn{2}{|c|}{$\begin{array}{l}\text { ARFID-Mixed } \\
\qquad(\mathrm{n}=10)\end{array}$} \\
\hline & Median (SD) & Range & Median (SD) & Range & Median (SD) & Range & Median (SD) & Range \\
\hline & & $(\min -$ & & (min - & & (min - & & $(\min -$ \\
\hline & & $\max )$ & & $\max )$ & & $\max )$ & & $\max )$ \\
\hline \multicolumn{9}{|l|}{ Assessment } \\
\hline \multirow[t]{2}{*}{ Age (years) } & $15.54(2.26)$ & $10.76-$ & 12.02 (1.09) & $11.77-$ & $15.17(2.66)$ & $9.49-17.51$ & 13.85 (1.99) & $9.82-16.63$ \\
\hline & & 17.43 & & 13.77 & & & & \\
\hline \multirow[t]{2}{*}{ BMI $\left(\mathrm{kg} / \mathrm{m}^{2}\right)$} & $16.03(2.23)$ & $11.78-$ & $15.82(1.01)$ & $14.43-$ & $14.86(2.13)$ & $11.71-$ & $14.48(2.70)$ & $12.63-$ \\
\hline & & 18.66 & & 16.40 & & 17.62 & & 20.55 \\
\hline \multirow[t]{2}{*}{ zBMI } & $-2.06(1.03)$ & $-3.75-$ & $-0.99(1.06)$ & $-2.62-$ & $-2.53(0.97)$ & $-4.05-$ & $-2.57(1.64)$ & $-3.79-1.11$ \\
\hline & & -0.64 & & -0.63 & & -1.27 & & \\
\hline HR (bpm) & $73.00(17.10)$ & $54-112$ & 77.00 (23.76) & $73-116$ & $88.00(25.56)$ & $49-130$ & 81.50 (18.63) & $45-111$ \\
\hline$\%$ TGW & $79.50(7.86)$ & $72-97$ & $84.00(9.54)$ & $83-100$ & $86.00(5.40)$ & $75-91$ & $86.00(6.93)$ & $79-100$ \\
\hline LOI (months) & $13.00(6.28)$ & $4-24$ & 129.00 & $84-144$ & $60.00(65.10)$ & $19-182$ & $86.00(68.68)$ & $3-177$ \\
\hline & & & $(31.23)$ & & & & & \\
\hline \multicolumn{9}{|l|}{ End of Treatment } \\
\hline Length of Treatment & 314.0 & $32-670$ & $693.0(41.01)$ & $664-722$ & 307.0 & 40-1001 & 425.50 & 169-804 \\
\hline (days) & (190.18) & & & & (297.62) & & (205.04) & \\
\hline \multirow[t]{2}{*}{ Age (years) } & 16.29 (1.95) & $12.59-$ & $14.67(1.52)$ & $13.59-$ & $15.68(2.24)$ & $12.23-$ & 15.03 (1.77) & $11.73-$ \\
\hline & & 18.30 & & 15.74 & & 18.04 & & 17.10 \\
\hline \multirow[t]{2}{*}{ BMI $\left(\mathrm{kg} / \mathrm{m}^{2}\right)$} & 19.33 (1.13) & $17.12-$ & $16.73(1.01)$ & $16.01-$ & $16.70(2.21)$ & $12.49-$ & $19.33(2.49)$ & $14.28-$ \\
\hline & & 20.65 & & 17.44 & & 19.11 & & 21.91 \\
\hline \multirow[t]{2}{*}{ zBMI } & $-0.69(0.52)$ & $-0.96-$ & $-1.42(0.90)$ & $-2.06-$ & $-1.40(1.17)$ & $-4.30-$ & $-0.45(1.16)$ & $-2.93-1.13$ \\
\hline & & 0.57 & & -0.78 & & -1.03 & & \\
\hline \% TGW & $100.00(7.39)$ & $81-100$ & $96.00(5.66)$ & $92-100$ & $97.50(6.14)$ & $82-100$ & $100.00(0.00)$ & $100-100$ \\
\hline
\end{tabular}

Notes. BMI = body mass index; zBMI = standardized body mass index by age and sex; HR = heart rate; \% TGW = percent of treatment goal weight; LOI $=$ length of illness prior to hospitalization.

Table 2. Sex, comorbid psychological frequencies and treatment objectives for pediatric ARFID patients ( $\mathrm{n}=26)$ admitted to a specialized pilot clinic. 


\begin{tabular}{|c|c|c|c|c|}
\hline & $\begin{array}{l}\text { ARFID-Aversive Hx } \\
\qquad(\mathrm{n}=6)\end{array}$ & $\begin{array}{l}\text { ARFID-Limited Variety } \\
\qquad(\mathrm{n}=2)\end{array}$ & $\begin{array}{l}\text { ARFID-Limited Intake } \\
\qquad(\mathrm{n}=8)\end{array}$ & $\begin{array}{l}\text { ARFID-Mixed } \\
\qquad(\mathrm{n}=10)\end{array}$ \\
\hline & No. (\%) & No. (\%) & No. (\%) & No. (\%) \\
\hline Female & $5(83.33 \%)$ & $1(50 \%)$ & $5(62.50 \%)$ & $4(40 \%)$ \\
\hline \multicolumn{5}{|l|}{ Comorbidities } \\
\hline Mood & $4(66.67 \%)$ & $1(50 \%)$ & $2(25 \%)$ & $3(30 \%)$ \\
\hline Anxiety & $5(83.33 \%)$ & $2(100 \%)$ & $3(37.50 \%)$ & $7(70 \%)$ \\
\hline Autism & $1(16.67 \%)$ & $2(100 \%)$ & $0(0 \%)$ & $4(40 \%)$ \\
\hline OCD & $2(33.33 \%)$ & $1(50 \%)$ & $0(0 \%)$ & $2(20 \%)$ \\
\hline ADHD & $2(33.33 \%)$ & $1(50 \%)$ & $1(12.50 \%)$ & $3(30 \%)$ \\
\hline Learning difficulties (has IEP) & $2(33.33 \%)$ & $0(0 \%)$ & $3(37.50 \%)$ & $4(40 \%)$ \\
\hline \multicolumn{5}{|l|}{ Treatment Objectives } \\
\hline Weight Gain & $6(100 \%)$ & $1(50 \%)$ & $8(100 \%)$ & $8(80 \%)$ \\
\hline Increasing Food Variety & $1(16.67 \%)$ & $2(100 \%)$ & $0(0 \%)$ & $6(60 \%)$ \\
\hline
\end{tabular}

Notes. OCD = obsessive compulsive disorder; ADHD = attention deficit hyperactivity disorder; IEP = individualized education program.

Table 3. Treatment modalities used for the management of pediatric ARFID patients $(n=26)$ admitted to a specialized pilot clinic. 


\begin{tabular}{|c|c|c|c|c|}
\hline & $\begin{array}{l}\text { ARFID-Aversive Hx } \\
\qquad(\mathrm{n}=6)\end{array}$ & $\begin{array}{l}\text { ARFID-Limited Variety } \\
\qquad(\mathrm{n}=2)\end{array}$ & $\begin{array}{l}\text { ARFID-Limited Intake } \\
\qquad(\mathrm{n}=8)\end{array}$ & $\begin{array}{l}\text { ARFID-Mixed } \\
\qquad(\mathrm{n}=10)\end{array}$ \\
\hline & No. (\%) & No. (\%) & No. (\%) & No. (\%) \\
\hline \multicolumn{5}{|l|}{ Treatment Modalities Utilized } \\
\hline Inpatient Admission & $3(50 \%)$ & $0(0 \%)$ & $1(12.50 \%)$ & $2(20 \%)$ \\
\hline Outpatient Therapy & $6(100 \%)$ & $2(100 \%)$ & $7(87.50 \%)$ & $9(90 \%)$ \\
\hline \multicolumn{5}{|l|}{ Therapy/ Treating Team Members } \\
\hline Individual Therapy & $4(66.67 \%)$ & $2(100 \%)$ & $2(25 \%)$ & $4(40 \%)$ \\
\hline Family Therapy & $4(66.67 \%)$ & $0(0 \%)$ & $1(12.50 \%)$ & $3(30 \%)$ \\
\hline Dietitian & $0(0 \%)$ & $1(50 \%)$ & $3(37.50 \%)$ & $7(70 \%)$ \\
\hline Occupational Therapist & $0(0 \%)$ & $2(100 \%)$ & $0(0 \%)$ & $1(10 \%)$ \\
\hline \multicolumn{5}{|l|}{ Medication } \\
\hline Atypical Antipsychotics & $3(50 \%)$ & $1(50 \%)$ & $1(12.50 \%)$ & $3(30 \%)$ \\
\hline Appetite Stimulants & $3(50 \%)$ & $1(50 \%)$ & $4(50 \%)$ & $3(30 \%)$ \\
\hline SSRIs & $3(50 \%)$ & $0(0 \%)$ & $2(25 \%)$ & $3(30 \%)$ \\
\hline Benzodiazepines & $2(33.33 \%)$ & $0(0 \%)$ & $0(0 \%)$ & $2(20 \%)$ \\
\hline Dopamine Receptor Antagonists & $2(33.33 \%)$ & $0(0 \%)$ & $0(0 \%)$ & $1(10 \%)$ \\
\hline SNRIs & $1(16.67 \%)$ & $1(50 \%)$ & $0(0 \%)$ & $0(0 \%)$ \\
\hline
\end{tabular}

Notes. SSRIs = selective serotonin reuptake inhibitors; SNRIs = serotonin-norepinephrine reuptake inhibitors.

Table 4. Treatment trajectories of pediatric ARFID patients $(n=26)$ admitted to a specialized pilot clinic.

\begin{tabular}{|c|c|c|c|c|}
\hline & $\begin{array}{l}\text { ARFID-Aversive } \\
\qquad(\mathrm{n}=6)\end{array}$ & $\begin{array}{l}\text { ARFID-Limited Variety } \\
\qquad(\mathrm{n}=2)\end{array}$ & $\begin{array}{l}\text { ARFID-Limited Intake } \\
\qquad(\mathrm{n}=8)\end{array}$ & $\begin{array}{l}\text { ARFID-Mixed } \\
\qquad(\mathrm{n}=10)\end{array}$ \\
\hline & No. (\%) & No. (\%) & No. (\%) & No. (\%) \\
\hline Treatment Reassessment Required & $4(66.67 \%)$ & $1(50 \%)$ & $4(50 \%)$ & $5(50 \%)$ \\
\hline Time to Reassessment (days) ${ }^{\mathrm{a}}$ & $17.00(42.19)$ & 104.00 & $377.00(181.07)$ & $32.00(107.13)$ \\
\hline Weight Restoration & $5(83.33 \%)$ & $1(50 \%)$ & $3(37.50 \%)$ & $8(80 \%)$ \\
\hline Restored at 6 Months & $3(50 \%)$ & $1(50 \%)$ & $2(25 \%)$ & $7(70 \%)$ \\
\hline Restored at 12 Months & $5(83.33 \%)$ & $1(50 \%)$ & $3(37.50 \%)$ & $8(80 \%)$ \\
\hline Time to Weight Restoration (days) ${ }^{\mathrm{a}, \mathrm{b}}$ & $138.00(114.26)$ & 104.00 & $193.00(145.66)$ & $111.00(130.56)$ \\
\hline
\end{tabular}


Notes. ${ }^{\mathrm{a}}$ Where applicable, values given are expressed as median (SD); ${ }^{\mathrm{b}}$ time to weight restoration was calculated based on patients who achieved weight restoration.

\section{Figures}

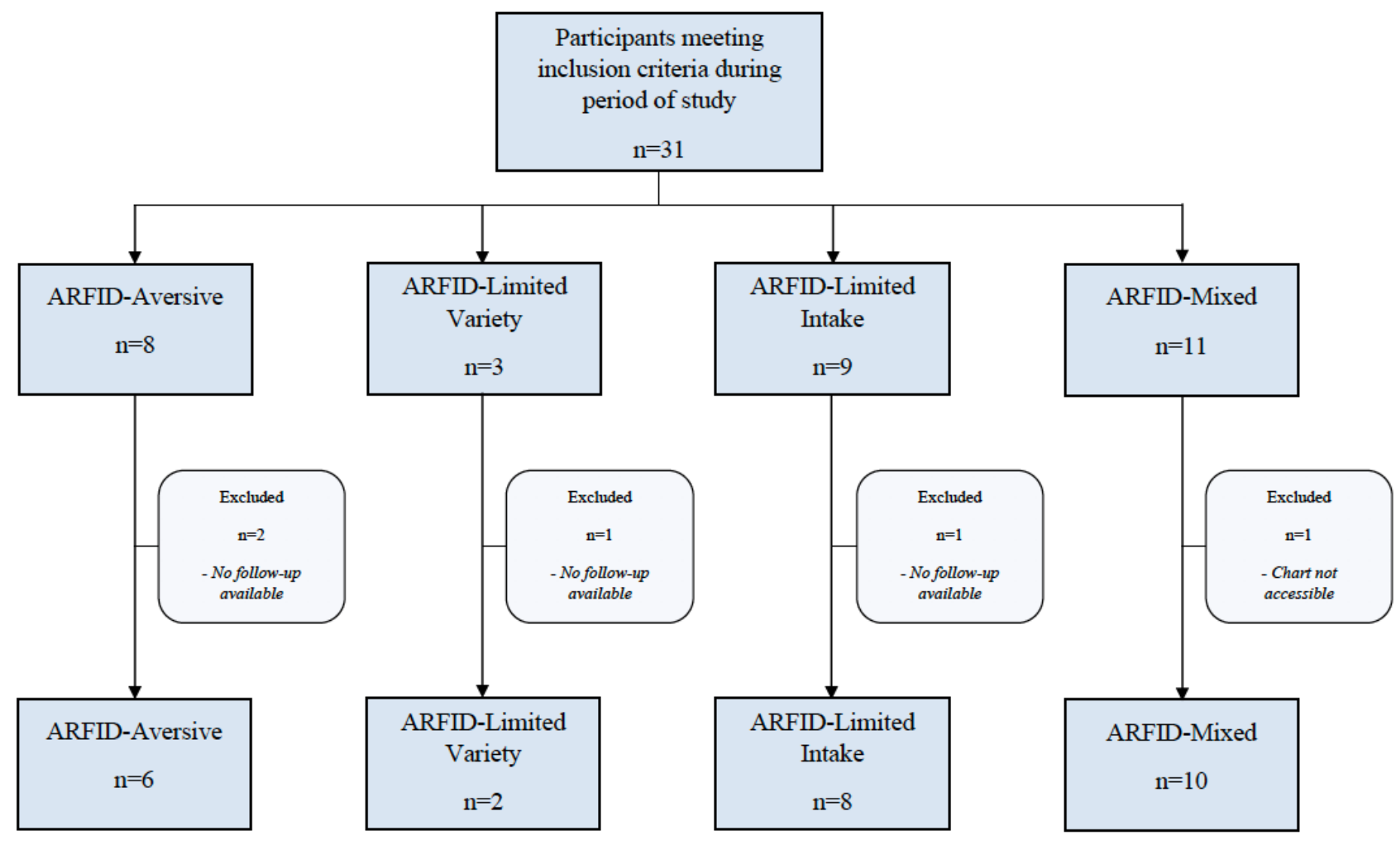

\section{Figure 1}

Flowchart of ARFID patients $(n=31)$ who were admitted to a specialized pilot clinic. 